\title{
World Health Day Theme-2013 and Role of Dental Profession
}

\section{Abhishek Mehta*}

Department of Public Health Dentistry, Faculty of Dentistry, Jamia Millia Islamia, New Delhi, India

World Health Organization (WHO) on the occasion of World health day i.e. $7^{\text {th }}$ April announces its health theme in order to focus attention of health professionals and authorities on one major public health problem threatening world population. Last year it has chosen condition of High Blood Pressure or commonly known as hypertension.

The reasons WHO chose to focus on hypertension are numerous, to name a few- this condition of high BP is bestowed with the name "SILENT KILLER" as it usually shows no symptoms in a person and the person slowly develops other associated illnesses. It affects over 1 billion people and it causes premature death of 9.4 million people every year worldwide. The problem is amplified by its increasing prevalence in lower and middle income countries as they encompass larger and increasing population [1].

In simple words, hypertension is defined as a systolic BP equal to or above $140 \mathrm{mmHg}$ and/or diastolic BP equal to or above $90 \mathrm{~mm} \mathrm{Hg}$. It's a non-communicable condition caused by behavior and lifestyle related factors such as eating too much sodium, being overweight, not exercising enough, stress, excessive alcohol and tobacco consumption. Prevention and control of high BP is therefore related to avoidance of above conditions/habits [1]. Other causes of Hypertension includes conditions like vitamin D deficiency, chronic kidney disease, adrenal or thyroid problems or tumors, drugs such as birth control pills and genetic factors. Early detection and management of high BP can reduce risk of cardio vascular problems like heart attack and failure, stroke and kidney failure.

Another area of concern is raising number of "prehypertensives" (systolic blood pressure 120-139 $\mathrm{mmHg}$ and/ or diastolic blood pressure $80-89 \mathrm{mmHg})$ in younger adults' population (20-35 years) around the world. Individuals with prehypertension have two-fold higher risk of mortality associated with stroke and coronary artery disease when compared with normotensives (individuals with BP less than 120/80 $\mathrm{mmHg}$ ). In addition, prehypertensives are at higher risk of developing hypertension and Cardio Vascular Diseases in their later lives [2].

Dental professionals can play an important role in early detection and referral of patients with hypertension and prehypertensives. During their practice, dentists come across significant number of patients with undetected high BP or uncontrolled hypertension and also known hypertensives. These patients are at high risk for various complications as well as for acute medical problems when receiving dental treatment [3]. A patient suffering from uncontrolled hypertension is usually refused any dental treatment especially surgeries till he/she get their blood pressure under control. Dentist must refer all such patients to their physician immediately.

It is usually observed that people visit their dentists more frequently than their physician especially when they are undergoing some rehabilitation work done in their oral cavity such as Root Canal Treatment, Dentures, periodontal surgery, implants etc. The dentists can utilize this opportunity to educate their patients about risk factors associated with hypertension. The dental professional can also check their BP at each appointment, this practice will help us in many ways, first of all we don't have to refer every known case of hypertension to the physician- if the BP is within normal range- before we start any dental procedure, and it will save our time. Secondly we might be able to discover undiagnosed cases of high blood pressure. Ultimately, in a long run, patients will think that you are not only treating their teeth but are concerned about their general health as well.

A person on anti hypertensive drugs can suffer from various oral manifestations such as xerostomia (dry mouth), gingival hyperplasia, dysguesia (taste alteration) and lichenoid reactions [4]. Fortunately there are many drug options available for controlling high BP, therefore, in most cases just by merely substituting the offending drug will reverse the side effect. The dentist must be aware of such manifestations and help its patients in making the right choice after consulting their physician.

Most of us whether living in developed or developing countries are dependent on primary health care model for our health and well being. This model effort to prevent, control and treat common communicable and non communicable diseases affecting the local population through its health centre's and various health promotional activities. Hypertension is one such condition which can be treated by a local physician/dentist sitting in a primary health centre.

Dental profession must come forward in reducing the prevalence of prehypertension and hypertension in their local population. This effort will not only benefit our patient but us also, as reduction in number of medically compromised patients will help us provide prompt treatment with minimal risk.

\section{References}

1. World Health Organization (2013) A global brief on hypertension-silent killer global public health crisis.

2. Pappachan MJ (2011) Increasing prevalence of lifestyle diseases: high time for action. Indian J Med Res 134: 143-145.

3. Hogan J, Radhakrishnan J (2012) The assessment and importance of hypertension in the dental setting. Dent Clin North Am 56: 731-745.

4. Little JW (2000) The impact on dentistry of recent advances in the management of hypertension. Oral Surg Oral Med Oral Pathol Oral Radiol Endod 90: 591599.

Corresponding author: Abhishek Mehta, Department of Public Health Dentistry, Faculty of Dentistry, Jamia Millia Islamia, New Delhi, India, Tel: +91-9971209069; E-mail: mehta_abhishek2003@yahoo.co.in

Received December 23, 2013; Accepted January 17, 2014; Published January 19, 2014

Citation: Mehta A (2014) World Health Day Theme-2013 and Role of Denta Profession. Dentistry 4: 192. doi:10.4172/2161-1122.1000192

Copyright: (c) 2014 Mehta A. This is an open-access article distributed under the terms of the Creative Commons Attribution License, which permits unrestricted use, distribution, and reproduction in any medium, provided the original author and source are credited. 